\title{
An EEG-Based Brain-Computer Interface for Dual Task Driving Detection
}

\begin{abstract}
The development of brain-computer interfaces $(\mathrm{BCl})$ for multiple applications has undergone extensive growth in recent years. Since distracted driving is a significant cause of traffic accidents, this study proposes one $\mathrm{BCl}$ system based on EEG for distracted driving. The removal of artifacts and the selection of useful brain sources are the essential and critical steps in the application of electroencephalography (EEG)-based $\mathrm{BCl}$. In the first model, artifacts are removed, and useful brain sources are selected based on independent component analysis (ICA). In the second model, all distracted and concentrated EEG epochs are recognized with a self-organizing map (SOM). This $\mathrm{BCl}$ system automatically identified independent components with artifacts for removal and detected distracted driving through the specific brain sources which are also selected automatically. The accuracy of the proposed system approached approximately $90 \%$ for the recognition of EEG epochs of distracted and concentrated driving according to the selected frontal and left motor components.
\end{abstract}

Keywords: Brain-Computer Interface (BCl); Electroencephalography (EEG); distracted driving; Independent Component Analysis (ICA); Self-Organizing Map (SOM)

\section{Introduction}

Numerous studies of human attention have confirmed that multiplexed information and tasks make focusing on driving difficult or impossible [1]. Safe automobile driving is a critical concern throughout the world, particularly when drivers are involved in multiple tasks that require watching for and reading road signs, tracking the locations of surrounding vehicles, judging when to pass others, navigating, and operating other technical equipment in the vehicle. These additional tasks can shift attention from driving and cause cognitive functions to compete for the brain resources required for safe driving, thus increasing the risk of traffic accidents. According to a statistical report on driving safety, distraction accounts for approximately $20-30 \%$ of traffic accidents [2]. Therefore, recognizing state of drivers' attention during driving is extremely critical.

Because the cognitive function of attention is directly related to competition for brain resources, the brain-computer interface $(\mathrm{BCl})$, which is based on the electroencephalogram (EEG), can be applied [3, 4]. Comparing with functional MRI (fMRI) and positron emission tomography (PET), EEG devices are more portable and have better time resolution. Several studies have also 
demonstrated that fluctuations in driving and distraction are accompanied by distinct EEG power spectrum changes recorded noninvasively from the scalp $[5,6]$. EEG signals are easily influenced by artifacts, and signals from different sources (sensors) are combined. This noise perturbation destroys or obscures the original characteristics of the signal, preventing the use of EEG signals in $\mathrm{BCl}$ applications. Therefore, artifact-free EEG data are the limiting factor for the performance of $\mathrm{BCl}$ systems. Signals with noise may become unusable; thus, signals with noise are not acceptable in any measurement system.

Most artifact-removal methods can be categorized into one of three types: (I) the error signal model is determined, and all similar models are then eliminated along the time course of the signal [7]; (II) high-frequency noises are removed by a low-pass filter according to the frequency course [8]; and (III) the original signal mixed with noise is separated into different instances [9-11]. Among these methods of artifact removal, independent component analysis (ICA) in the third type of method performs better than other methods [12]. The aim of this study was to remove artifacts from EEG signals with an ICA-based method.

After applying the ICA algorithm to the recorded EEG signals, the artifact components need to be rejected manually by experts [13], and the remaining components represent useful brain sources. There are two drawbacks: first, the results fully depend on the availability of experts, and the process of analyzing these selected independent components is subjective. The analysis of some ambiguous components would likely vary according to the experts' judgment. Second, the purpose of many $\mathrm{BCl}$ applications is online detection, which would not benefit from this type of manual selection method. Our previous studies prompted the development of a method to remove artifact components to minimize the subjective variance [3]. For various applications of the $\mathrm{BCl}$ system, the specific component features are also important. The useful spatial brain components also must be identified and selected for advanced analysis according to the spatial locations of the sources. Distinct brain functions can be separated by spatial brain regions. Then the problems could be solved with our automated expert system of component selection for useful brain sources.

In this investigation, two models were combined as one $\mathrm{BCl}$ system, as shown in Fig. 1. The first model automatically rejects artifact brain signals and selects the useful features of specific independent components. In the second model, these selected components are then applied to identify signatures of distracted or concentrated driving. 


\section{Materials}

The procedures used to collect data and the main algorithms used in this investigation are described in this section.

\subsection{Experiment and participants}

Driving a car on an actual road while performing another task is extremely dangerous. Our experiment was performed in a surrounded virtual reality (VR)-based driving environment, which consisted of surrounding highway driving scenes produced using seven projectors and a real vehicle mounted on a Stewart platform with six degrees of freedom (6-DOF). The four-lane road in the VR scene had a median strip in its center. Two designed tasks were implemented to investigate distracted driving: an unexpected deviation (swerving) of the car and the presentation of mathematical equations. In the deviation task, the car randomly deviated left or right from the third lane. This task required participants to compensate for this deviation using the steering wheel to keep the car in the center of the third (from left to right) cruising lane. Two-digit addition mathematical equations were presented to the subjects in the mental calculation task. The answers to all equations were given and were either right or wrong. The subjects had to press corresponding buttons on the steering wheel as rapidly as possible. When the equation was correct, the subjects had to press the right button. Conversely, the subjects pressed the left button to indicate an incorrect mathematics equation. The allotment ratio of correct-incorrect equations was 50-50.

Both of these tasks and the stimulus onset asynchrony (SOA) were considered to provide different distraction levels [14], and five conditions were developed as described below.

(I) Case-1: The mathematical equation is presented 400 ms earlier than the occurrence of the deviation (MID).

(II) Case-2: The car deviation and mathematical equation occur simultaneously (D\&M).

(III) Case-3: The car deviation is 400 ms earlier than the presentation of the mathematical equation (DIM).

(IV) Case-4: Only the mathematical equation is presented (M).

(V) Case-5: Only the car deviation occurs (D).

These five cases (conditions) were presented randomly to avoid anticipation [15]. The inter-trial intervals were set from six to eight seconds, and the independent trials did not interact to avoid affecting the subject. Thus, as many as 100 trials were presented to the subject in each session to ensure that the 
number of events was adequate for statistical analysis. Approximately 80 trials of each of the cases outlined above were performed in an entire experiment.

Fourteen volunteers (average age 26.2 years) currently studying at National Chiao Tung University participated in this experiment. All participants had a valid driver's license, with a mean reported driving experience of 5 years. Each subject was free of neurological and psychological disorders as well as drug and alcohol abuse. Participants practiced for approximately 15 minutes to avoid the learning effect [16]. The subjects were also asked to pay full attention to driving while reacting quickly to each type of task. Therefore, the subjects were obliged to rest after driving for 15 minutes to avoid the impact of fatigue. Scalp EEG signals were recorded with 32 channels following the 10-20 international system of electrode placement. Before acquiring EEG data, the contact impedance between the EEG electrodes and the skin was calibrated to less than $5 \mathrm{k} \Omega$ by injecting a $\mathrm{NaCl}$-based conductive gel. The EEG data were recorded with 16-bit quantization levels at a sampling rate of $1000 \mathrm{~Hz}$.

\subsection{Independent Component Analysis}

Independent component analysis (ICA) is a computational method for separating multi-source signals into subcomponents, with the assumption that the signals are mutually statistically independent $[17,18]$. This analysis is one type of blind source separation that determines the independent components by maximizing the statistical independence of the estimated components. Segregating, identifying, and localizing EEG sources are extremely difficult because EEG data collected the brain activities within large brain areas from the human scalp. A prior study [10] first applied ICA to biomedical time series data (EEG data). In ICA, it is assumed that the aggregation of signals from different brain sources is linear and instantaneous (no time delay). Although the conductivity between the skull and brain varies, it does not cause a significant time delay. Therefore, ICA effectively isolates electrical activity recorded from all individual EEG channels [10, 11, 19], and sources with artifacts can be detected and removed easily [19, 20].

ICA models the data using the equation:

$$
u=W X
$$

where $X$ is the data recorded from individual EEG channels, $W$ is a weight matrix for projecting the mixing independent components back into original signals, and $u$ is the un-mixing matrix. The inverse weight matrix, $W^{1}$, is then computed, and the useful components are selected. The projection of the $i^{\text {th }}$ independent component onto the original data channels is then given by 


$$
X_{\text {clean }}(i)=W^{1}(:, i)^{*} u(i,:)
$$

where each matrix column, $W^{-1}$, indicates the activation weights distributed across the electrodes for each independent component, which can be rendered as a two-dimensional (2D) topographic map on the scalp.

After applying ICA, the multi-channel EEG signal is separated according to several independent brain sources. If there are $\mathrm{N}$ sensors, $\mathrm{N}$ sources are separated with the ICA algorithm [10]. The components must first be judged to be useful(good) or artifact(bad) brain components. Bad components may include bad channels or artifacts such as eye-blinking, muscle noise, heart signals, line noise, breathing noise, or environmental factors [11]. Such components are always present and influence the raw EEG signal. The selected useful(good) components may then be classified according to distinct spatial regions to analyze specific brain signals. The specific components measured are the frontal, central, parietal, occipital, and motor components. The occipital and motor component can be spatially isolated to left and right areas. In this study, the left and right occipital components were all regarded as occipital components.

\subsection{Self-Organizing Map}

A self-organizing map (SOM) is an unsupervised neural network [21]. The learning process of the SOM is similar to the information representation properties of many functions of the brain [22]. The SOM is an unsupervised cluster algorithm that provides mechanisms for visualizing the complex distribution of cognitive states. This algorithm has been widely used in various research areas [23-25]. High-dimensional data are projected to low-dimensional data and then easily analyzed through their topographic organization. Dimension projection in the SOM algorithm is achieved using two layers: the input layer and the output layer.

In the input layer, each neuron in the map contains an $n$-dimensional (as in the input data) reference vector. In each learning process, the Euclidean distance is computed between the training sample and the neuron. The training sample is projected to the neuron with the shortest distance. This neuron is stimulated to adapt to the projected training sample. Competitive learning is an essential characteristic of the SOM algorithm. The chosen neuron and its neighbor neurons are adapted. The degree of adaption depends on the distance between the two neurons.

In the output layer, topological neighborhoods can be of different shapes, such as a rectangle or a hexagon. $K$ neurons (locations) are arranged as 
lattices with visual dimensions $(1 \mathrm{D}, 2 \mathrm{D}$, or $3 \mathrm{D})$, where $K$ is the number of neurons on the map. During the training process, similar data are projected on the neighbor neurons to cluster the main areas.

When the unsupervised training process is complete, the topographic organization of the map adequately represents the distribution of the data. The growth of a child involves numerous types of stimuli, and neurons with similar functions are clustered in the same brain areas. Therefore, the SOM imitates the learning mechanism of the brain. This property of the SOM was used to investigate the EEG signals. We applied the SOM to train the maps and analyze the relationships among data. We also created classifiers with the trained map to recognize unknown data. In this study, the maps were initialized, trained, and evaluated with the SOM toolbox for MATLAB [26].

\section{Methods}

Two proposed models were combined in our $\mathrm{BCl}$ system. The useful brain sources were selected in model 1 , and these extracted brain sources were then analyzed in model 2. The details of these two models are described in the following sections.

\subsection{Model 1: Automatically selecting useful brain sources}

A hierarchical model capable of automatically selecting components was proposed, as shown in Fig. 2. Unlike previous methods, our model can identify useful components and recognize the spatial components from the remaining useful components for advance analysis. The training data used to construct the automatically selecting model were collected during our previous experiments and included 25 subjects and 700 independent components.

\subsubsection{Data Processing}

The EEG data were recorded at a 16-bit quantization level and a sampling rate of $1000 \mathrm{~Hz}$. In data pre-processing, the recordings were down-sampled to a rate equal to $250 \mathrm{~Hz}$ to simplify data processing. The EEG data were then processed for further analysis with a simple low-pass filter with a cut-off frequency of $50 \mathrm{~Hz}$ to remove the line noise $(60 \mathrm{~Hz}$ and its harmonics) and other high-frequency noise. The DC drift was also removed by applying a simple high-pass filter with a cut-off frequency of $0.5 \mathrm{~Hz}$. Because we designed different cases by combining the driving and mathematics tasks, the EEG responses related to different cases were extracted from the original EEG signals for further analysis. The EEG epochs were extracted from continuous EEG data and combined under the same conditions as those used for the ICA. 
Although the EEG data were collected with 32 electrodes, the two reference channels needed to be removed. The electrodes Fp1 and Fp2 were located close to the eyes, and eye movement easily influences the raw EEG signal; hence, these two channels were also removed to reduce the interference caused by eye blinking. The ICA identified 28 independent components for each subject. To eliminate the differences between individuals, we normalized the weight matrix using the z-score method. Each component was labeled as useful or non-useful (artifact) by several experienced experts capable of interpreting and analyzing EEG data to label each scalp map. The automatically selecting model was finally clustered all useful components to the distinct brain areas.

\subsubsection{Component identification}

In the first layer of selecting components, the goal is to automatically identify useful or artifact components to solve the two-class problem. The basic support vector machine (SVM) is formulated for a two-class problem [27, 28]. If the training data are linearly separable, the SVM then finds an optimal hyperplane that maximizes the margin of separation between the two classes. The kernel of radial basis function (RBF) is usually used; therefore, the SVM with RBF kernel is called the SVMRBF. All training data were equally divided into 10 parts to apply 10 -fold cross-validation. In each fold, one part was chosen to be the testing data, and the others became the training data. These nine parts were trained with the SVMRBF to obtain one classifier, and this trained classifier was verified with the testing data. This procedure was repeated 10 times to collect 10 classifiers with different training data. These 10 classifiers were then combined into one classifier according to a majority vote to identify useful or artifact components.

At the second layer, the goal is to identify specific brain regions using the artifact-free useful components identified in the first layer. As with the previous data, these data were also collected from the 25 subjects. There were 115 useful components after automatic artifact removal by the first layer, and detailed numbers of each type of independent component are shown in Table I. The components represent different sources according to the spatial relationship of the brain. We then applied the SOM to classify these spatial components. After classification and identification by this automatic selecting model, the artifact-free components were assigned to one of six spatial brain regions.

\subsection{Model 2: Detecting distracted driving}


According to model 1 , the frontal, central, parietal, left, and right motor components were selected to analyze the signature of driver distraction. We investigated these five selected components through the SOM. The frontal and left motor components were then selected as the main brain sources for detecting distracted driving.

\subsubsection{Feature extraction}

Before we could analyze the signature of distracted driving from the extracted component data using the SOM, the features of these components had to be extracted. The factor of stimulus onset asynchrony (SOA) was considered in this experiment, particularly regarding the different time intervals of the dual tasks. We designed the epoch-based experiment to ensure that the features were extracted based on the epoch. The EEG epoch was related to the event or stimulus in the experiments. The length of each EEG epoch in this distraction experiment was five thousand milliseconds, which comprises the baseline, totaling one thousand milliseconds, and the phasic portion, totaling four thousand milliseconds.

In the pre-processing steps, the sampling rate was reduced to $250 \mathrm{~Hz}$; hence, one epoch yielded 1250 data samples. To obtain more information, a fast Fourier transform (FFT) was applied to transform the EEG signals from the time domain to the frequency domain. Because the SOA factor was considered in this experiment, the time interval was set to $400 \mathrm{~ms}$. Both the frequency information and the time information were preserved. The phasic portion of the EEG epoch was divided equally into 10 intervals with lengths of $400 \mathrm{~ms}$. A FFT was then applied to the EEG signal in each interval (baseline and 10 phasic intervals) to compute the perturbation of the frequencies. The time and frequency information provide higher quality features when FFT is applied. There were 550 data points in one EEG epoch ( $50 \mathrm{~Hz}^{*} 11$ intervals). To reduce the dimensions and obtain better descriptions of the EEG epochs, only those data points from $1-30 \mathrm{~Hz}$ were considered. Therefore, a total of 330 dimensions were extracted in each EEG epoch.

The EEG signal that occurred one second before the onset of the event was defined as the baseline in this study. To investigate the changes in spectral power and the perturbations in the oscillatory dynamics of the ongoing EEG, the baseline of each EEG epoch was removed to ensure that the EEG signals were primarily caused by reacting to the designed tasks and not by the "state of resting". A complete experiment consisted of four sessions. Each session encompassing all trials was conducted under the same circumstances, and the subjects maintained the same psychological and physical states throughout 
the experiments. However, different subjects were not necessarily given the same phenomena for the same task; in other words, the degree of spectrum power or mental state for the same tasks were not the same for all participants. We therefore analyzed the influence of distraction on each participant instead of the differences among all subjects. The diversity and variation among all subjects must be minimized because the goal of this study was to provide a general system for detecting distracted driving. The mean value and standard deviation were computed using the EEG data collected from one specific subject, and the EEG signal was then normalized using the Fisher z-score method to reduce the variation among subjects. After feature processing, each EEG epoch contained 300 data points. Five independent components (frontal, central, occipital, right motor, and left motor) were selected through our automatic system, as described previously. The EEG signals in each individual component were trained using the SOM to analyze the signature of distracted driving.

After considering the performance (as shown in the results section) and the number of subjects, the EEG signals from the frontal and left motor components were selected as the features of distracted driving for our $\mathrm{BCl}$ system in this study. Previous studies have demonstrated that the frontal and motor components are related to the characteristics of distracted driving [5]. Activation in the frontal areas is induced by performing a mental task $[29,30]$. The power changes in the frontal area primarily reflect the activation of neurons involved in allocating attention to the stimuli of different tasks [31]. The spectra in the motor component were selected because the subjects had to react to either the car deviation or a mathematics equation [32]. The power changes of the motor component during motor planning are involved in preparing for steering the wheel and answering the math questions. Therefore, for the input data (feature), the characteristics of the frontal and the left motor components were combined as one complete feature. The main phenomenon in the frontal area is reported to be approximately 1 to $20 \mathrm{~Hz}$; hence, the feature in the frontal component was reduced from 300 dimensions to 200 dimensions [5]. Then the dimension of the combined features was 500 (frontal: 200 and motor: 300). The same parameters used in the SOM were applied to trained maps for the distracted driving detection system.

\subsubsection{Detecting the signature of distracted driving}

This study proposes a model for detecting distracted driving. Based on the EEG data from two selected components, the leave-one-out method was considered in this simulation, as shown in Fig. 3. Classification in our model 
involved conducting a majority vote according to the different classifiers, which were nine trained maps [33]. The EEG data of a chosen subject were the testing data, and the other EEG epochs of the remaining subjects were used as the training data. Therefore, the training data were collected from 10 subjects. Nine maps were then generated using this training data with the same SOM parameters used previously. Unlabeled neurons on the map were labeled first through the shortest distance from the neighboring labeled neurons, with the unlabeled neuron given the same label as the specifically labeled neighboring neuron. All fully labeled maps were then considered to provide complete information about the relationships among the neural neighborhood. These nine trained and fully labeled maps were used as the dataset to verify the testing data by majority voting. Each EEG epoch belonged to one best matching neuron with the shortest Euclidean distance for each map. We obtained nine results using the database and classified the EEG epoch by voting. The class with the highest frequency was assigned to that EEG epoch. This simulation was repeated 11 times for the EEG epoch of each subject.

\section{Results}

\subsection{Component identification}

The independent components collected from 14 subjects were the testing data for the automatically selecting components model, and there were 392 $\left(14^{\star} 28\right)$ independent components in this testing data. Fig. 4(a) depicts the useful and artifact components that were separated from one subject. In Fig. 4(b), the selected useful components are labeled according to the spatial brain sources. The useful components were selected in the first layer in model 1. The labeling accuracy is shown in Table 2, with an average value of $90 \%$. In this layer, all bad components were accurately identified, while the useful components were occasionally erroneously classified as bad components. Therefore, perfect classification of useful brain sources remains a challenge. The number of useful components for each subject is also shown in Table 2. These useful components were classified again into different spatial brain areas by the second layer.

There were 115 useful independent components collected from the previous 25 subjects with 700 components, as shown in Table 1. These data were used to create one classifier for identifying different spatial brain sources. This classifier is one 2-D map, as shown in Fig. 5. There are six perfect clusters representing six brain sources on this map. The labeling accuracy using this 
map was $100 \%$ for each brain source. Using this map, the SOM was able to represent the spatial differences between brain sources, and the variations caused by subjective judgment were reduced. The left and right motor components were projected to the neighbor neurons. There are also spatial characteristics among the neurons representing the frontal, central, and parietal components. Although the occipital components are located in the middle of this map, they are still near the parietal, left, and right motor components. Therefore, the relationships between different independent components could be discovered using the SOM. The map shown in Fig. 5 can also be used as a classifier to identify distinct brain sources. The selected useful components with different brain sources in Table 2 were then identified using this map. These useful components were classified among different spatial brain sources, as shown in Table 3. The accuracy of the classification was $100 \%$ for each selected type of component (spatial brain sources).

To automatically identify components, clustering components are also provided in EEGLAB, a popular toolbox used to analyze EEG data. Using this toolbox, the mean accuracy of each type of spatial component was $95.6 \%$. The results of this method let some components be classified to other clusters in adjacent areas. If the variance of misclassification is reduced, the analysis of EEG signals will be more stable. By using our automatic model, each component was recognized successfully with high accuracy and low variance Therefore, the SOM is a better classifier than the available method in EEGLAB to identify distinct spatial brain sources.

We collected data from 14 subjects in this experiment, but the number of spatial brain sources was less than 14; if a particular brain area was not highly activated when the subjects performed specific tasks, then that area or component could not be separated in the ICA results. Since the frontal cluster contains the largest number of brain sources, the number of this component reaches to 14 as Table 3 . So the frontal region may be the most important component in distracted driving. As previously mentioned, the frontal component is known to be highly associated with attention-demanding tasks [31]. When an individual drives or responds to other tasks, their motor areas may be involved in planning the motions [32]. In agreement with this result, the number of left and right motor areas was identified as 11 and 10 in Table 3, respectively.

\subsection{The signature of distracted driving in distinct brain sources}

Six types of components were identified using the previous automatic selecting system. Here, the selected components from the 14 subjects were 
used to analyze the differences in EEG epochs during distracted driving in the $\mathrm{BCl}$ system. The occipital components can be divided into bilateral and backward parts, and the number of identified components in these two clusters is too low to be used. So EEG epochs were collected from the frontal, central, parietal, left motor, and right motor components were considered except the occipital component. The trained maps are depicted in Fig. 6, and Table 4 lists the correct labeling percentage. In these five maps, the neurons represent single conditions that were clustered more accurately than those of the other three dual conditions. The EEG epochs of dual conditions were usually projected to particular sub-areas, indicating that the differences among the brain activity phenomena in one situation were discovered using a single type of component, which may not represent the effects of distracted driving.

When examining these maps according to the labeling accuracy and distribution of neurons, the frontal, left motor and right motor components were selected for the index of distracted driving for two reasons. First, the accuracy of the three maps in these components was the highest, with an average accuracy of more than $85 \%$. This indicates that each component could represent the differences among the five conditions. Second, the distribution of labeled neurons was more accurately clustered. SOM analysis involves projecting similar data to neighbor neurons and the relationships among the data can be illustrated according to the distribution of each neuron on the well-trained map. The maps of the left motor and frontal components are clearly clustered to the EEG signals collected from single conditions, and the neurons of dual conditions are also clustered to several small groups. Because the results of the left motor component are more favorable than those of the right motor component, the right motor component was discarded. Therefore, all EEG epochs in the frontal and motor components are considered the effective indices of distracted driving.

Fig. 7 shows the trained maps with complex features, and the accuracy of these two maps is given in Table 5, which shows the average rate of correct labeling. The features of the frontal and motor components are combined to provide more detailed information for detecting distracted driving because Case-2 is usually divided into sub-groups that can be as small as a single neuron. Under this condition, our designed tasks appeared simultaneously, and the subjects were asked to perform them well. Their brain resources may have competed and been activated by two visual stimuli [34]. The EEG epochs of Case-2 were excluded first. The map shown in Fig. 7(a) was trained using the EEG epochs of only four conditions. The labeling accuracy was also higher 
than $95 \%$. Four areas, indicated by different colors on this map, were discovered. In particular, the EEG epochs from two single conditions (Case-4 and Case-5) are clustered accurately to two almost-perfect areas in the corner of this map. Although some neurons representing dual conditions (Case-1 and Case-3) are clustered into small sub-groups, two main clusters are still found. Fig. 7(b) depicts the map trained using all EEG epochs from the five conditions. The phenomena of the other four cases are similar to the results shown in Fig. $7(a)$, while the neurons labeled as Case- 2 are separated into several sub-groups on this map, without clustering into one main area similar to the other four classes. According to the results of classifying the EEG epochs of these five cases, the results of Case-2 represent the lowest labeling accuracy and the largest standard deviation.

The EEG could be one source for distinguishing distracted driving, according to the results of the combined features. There is a noticeable difference in the EEG spectra between distracted and undistracted drivers. When the subjects faced only a single stimulus, either car deviation or a mathematical equation, their brain resources were fully allocated to these tasks. In the map results, the EEG epochs of two single conditions were consistent and clustered accurately to two perfect areas. By contrast, when the subjects performed two tasks within two seconds (by behavior analysis), they may have been distracted from driving by the secondary task of answering the mathematical equation. When two tasks occurred simultaneously, the subjects employed different strategies to respond to them. They may have chosen one task to answer first and then completed the other task, in which case the EEG phenomenon was similar to that of either Case-1 or Case-3. They may have neglected one task to react perfectly to the other, in which case the EEG epochs were the same as those of single conditions. This is the reason for the disorderly neurons of Case-2. According to our SOM maps in Fig. 7, brain activations could indeed represent the effects of distracted driving.

Several studies have evaluated the feasibility of classifying EEG data for extensive applications. Simple logic functions, linear or nonlinear regression, and neural networks have been used for this purpose [35-39]. The classification performance is almost $90 \%$. Using the linear method, the EEG epochs could not be recognized clearly, and the performance was approximately $42.6 \%$. Nonlinear algorithms were also considered. The average accuracy of our data reached $63.6 \%$ using a SVM with a radial basis function. In [38], the use of SVMs for automatic EEG classification was proposed. In [39], SOM was used to classify EEG patterns. SOM not only 
recognizes differences but also provides a visual map to represent the relationships among the data. In the present study, an accuracy of approximately $90 \%$ was obtained with the SOM.

\subsection{Testing performance}

The SOM could distinguish differences between brain activities with high accuracy under all five conditions. Case-5 is defined as concentration driving because the subjects only controlled the steering wheel when responding to car deviation. The subjects were required to analyze mathematical equations under the other four conditions (Case-1 - Case-4), causing their attention to shift from driving to the secondary task. Hence, those four conditions were defined as distracted driving. Our brain-computer interface system was able to identify the phenomenon of EEG signals as distracted or concentrated driving, and the result of identification by our $\mathrm{BCl}$ was transformed into a two-class problem. Each piece of testing data was a new EEG signal from one subject that was not included in the training data. The performance rates for detecting driver distraction with the SOM are shown in Table 6 . The hit rates of distracted and concentrated driving were $84.1 \%$ and $91.5 \%$, respectively. Therefore, this system can recognize the EEG epochs of one new subject and assign them to one of two clusters: a concentrated or distracted driver.

\section{Conclusions}

In this study, an automatic detection system for distracted driving was proposed; this system involves the selection of components with artifact removal and judgments of driver distraction levels. The useful components are extracted to provide more effective information. Based on the selected useful components, the automatic classification model with the SOM can clearly classify six different spatial brain sources, including frontal, central, parietal, right motor and left motor components, for advanced analysis. The feature composed of the selected frontal and left motor components is novel and useful for detecting distracted driving. Considering the frontal and left motor components, the proposed $\mathrm{BCl}$ system reached a maximum accuracy of approximately $90 \%$ for the recognition of EEG epochs of distracted and concentrated driving. Therefore, the frontal and left motor components are the main areas that respond to multiple tasks during distracted driving. The recognition of distraction levels could be used to monitor drivers and warn them to pay more attention to avoid traffic accidents in real-life driving. 


\section{ACKNOWLEDGMENTS}

This work was supported by the UST-UCSD International Center of Excellence in Advanced Bio-engineering, sponsored by the Taiwan National Science Council I-RiCE Program under Grant Number: NSC-100-2911-I-009-101, by the National Science Council, Taiwan, under Contract NSC 100-2321-B-009-003, and by the Aiming for the Top University Plan, under Contract 100W9633.

\section{References:}

[1] S.J. Kass, K.S. Cole, C.J. Stanny, Effects of distraction and experience on situation awareness and simulated driving, Transport Res F-Traf, 10 (2007) 321-329.

[2] K. Young, M. Regan, I.J. Faulks, M. Stevenson, J. Brown, A. Porter, J.D. Irwin, Driver distraction: A review of the literature, Distracted Driving, (NSW: Australian College of Road Safety, Sydney, 2007), pp. 379-405.

[3] C.T. Lin, L.W. Ko, T.K. Shen, Computational intelligent brain computer interaction and its applications on driving cognition, Computational Intelligence Magazine, IEEE, 4 (2009) 32-46.

[4] N.R. Pal, C.Y. Chuang, L.W. Ko, C.F. Chao, T.P. Jung, S.F. Liang, C.T. Lin, EEG-Based Subject- and Session-independent Drowsiness Detection: An Unsupervised Approach, Eurasip J Adv Sig Pr, 2008 (2008) 1-11.

[5] C.T. Lin, S.A. Chen, T.T. Chiu, H.Z. Lin, L.W. Ko, Spatial and temporal EEG dynamics of dual-task driving performance, Journal of NeuroEngineering and Rehabilitation, 8 (2011) 1-11.

[6] S. Lei, M. Roetting, Influence of task combination on EEG spectrum modulation for driver workload estimation, Hum Factors, 53 (2011) 168-179.

[7] A. Erfanian, B. Mahmoudi, Real-time ocular artifact suppression using recurrent neural network for electro-encephalogram based brain-computer interface, Medical \& biological engineering \& computing, 43 (2005) 296-305.

[8] P. He, G. Wilson, C. Russell, Removal of ocular artifacts from electro-encephalogram by adaptive filtering, Medical \& biological engineering \& computing, 42 (2004) 407-412.

[9] T.D. Lagerlund, F.W. Sharbrough, N.E. Busacker, Spatial filtering of multichannel electroencephalographic recordings through principal component analysis by singular value decomposition, Journal of Clinical Neurophysiology, 14 (1997) 73-82. [10] S. Makeig, A.J. Bell, T.P. Jung, T.J. Sejnowski, Independent component analysis of electroencephalographic data, Adv Neur In, 8 (1996) 145-151.

[11] T.P. Jung, S. Makeig, C. Humphries, T.W. Lee, M.J. McKeown, V. Iragui, T.J. 
Sejnowski, Removing electroencephalographic artifacts by blind source separation, Psychophysiology, 37 (2000) 163-178.

[12] D. Mantini, M.G. Perrucci, S. Cugini, A. Ferretti, G.L. Romani, C. Del Gratta, Complete artifact removal for EEG recorded during continuous $\mathrm{fMRI}$ using independent component analysis, Neurolmage, 34 (2007) 598-607.

[13] T.P. Jung, S. Makeig, M. Westerfield, J. Townsend, E. Courchesne, T.J. Sejnowski, Analysis and visualization of single-trial event-related potentials, Hum Brain Mapp, 14 (2001) 166-185.

[14] F. Verbruggen, A.R. Aron, M.A. Stevens, C.D. Chambers, Theta burst stimulation dissociates attention and action updating in human inferior frontal cortex, Proceedings of the National Academy of Sciences of the United States of America, 107 (2010) 13966-13971.

[15] K.I. Erickson, S.J. Colcombe, R. Wadhwa, L. Bherer, M.S. Peterson, P.E. Scalf, A.F. Kramer, Neural correlates of dual-task performance after minimizing task-preparation, Neurolmage, 28 (2005) 967-979.

[16] T.A. Kelley, S. Yantis, Learning to attend: effects of practice on information selection, Journal of Vision, 9 (2009) 1-18.

[17] C. Jutten, J. Herault, Blind separation of sources, part I: An adaptive algorithm based on neuromimetic architecture, Signal Processing, 24 (1991) 1-10.

[18] A. Hyvarinen, E. Oja, Independent component analysis: algorithms and applications, Neural networks : the official journal of the International Neural Network Society, 13 (2000) 411-430.

[19] T.W. Lee, M. Girolami, T.J. Sejnowski, Independent component analysis using an extended infomax algorithm for mixed subgaussian and supergaussian sources, Neural Computation, 11 (1999) 417-441.

[20] V. Ricardo Nuno, Extraction of ocular artefacts from EEG using independent component analysis, Electroencephalography and Clinical Neurophysiology, 103 (1997) 395-404.

[21] T. Kohonen, The self-organizing map, Proceedings of the IEEE, 78 (1990) 1464-1480.

[22] T. Kohonen, Self-organization and associative memory: 3rd edition, Springer-Verlag New York, Inc., 1989).

[23] T. Kohonen, P. Somervuo, Self-organizing maps of symbol strings, Neurocomputing, 21 (1998) 19-30.

[24] J. Vesanto, E. Alhoniemi, Clustering of the self-organizing map, IEEE transactions on neural networks / a publication of the IEEE Neural Networks Council, 11 (2000) 586-600.

[25] T. Kohonen, E. Oja, O. Simula, A. Visa, J. Kangas, Engineering applications of the 
self-organizing map, Proceedings of the IEEE, 84 (1996) 1358-1384.

[26] J. Vesanto, J. Himberg, E. Alhoniemi, J. Parhankangas, S. Team, L. Oy, Som toolbox for matlab, Techn. Ber., Helsinki University of Technology, (2000).

[27] V.N. Vapnik, The nature of statistical learning theory (Springer, 2000).

[28] C. Cortes, V. Vapnik, Support-Vector Networks, Machine Learning, 20 (1995) 273-297.

[29] H. Asada, Y. Fukuda, S. Tsunoda, M. Yamaguchi, M. Tonoike, Frontal midline theta rhythms reflect alternative activation of prefrontal cortex and anterior cingulate cortex in humans, Neurosci Lett, 274 (1999) 29-32.

[30] E.K. Miller, J.D. Cohen, An integrative theory of prefrontal cortex function, Annu Rev Neurosci, 24 (2001) 167-202.

[31] P. Missonnier, M.P. Deiber, G. Gold, P. Millet, M. Gex-Fabry Pun, L. Fazio-Costa, P. Giannakopoulos, V. Ibanez, Frontal theta event-related synchronization: comparison of directed attention and working memory load effects, J Neural Transm, 113 (2006) 1477-1486.

[32] M.M. Hayhoe, A. Shrivastava, R. Mruczek, J.B. Pelz, Visual memory and motor planning in a natural task, Journal of Vision, 3 (2003) 49-63.

[33] C.T. Lin, Y.K. Wang, S.A. Chen, An EEG-Based Brain-Computer Interface for Dual Task Driving Detection, in: B.L. Lu, L. Zhang, J. Kwok (Eds.) Neural Information Processing, (Springer Berlin / Heidelberg, 2011), pp. 701-708.

[34] H. Deubel, W.X. Schneider, Saccade target selection and object recognition: evidence for a common attentional mechanism, Vision Research, 36 (1996) 1827-1837.

[35] K.Q. Shen, X.P. Li, C.J. Ong, S.Y. Shao, E.P. Wilder-Smith, EEG-based mental fatigue measurement using multi-class support vector machines with confidence estimate, Clin Neurophysiol, 119 (2008) 1524-1533.

[36] G. Townsend, B. Graimann, G. Pfurtscheller, Continuous EEG classification during motor imagery--simulation of an asynchronous $\mathrm{BCl}$, IEEE transactions on neural systems and rehabilitation engineering : a publication of the IEEE Engineering in Medicine and Biology Society, 12 (2004) 258-265.

[37] F. Faradji, R.K. Ward, G.E. Birch, Toward development of a two-state brain-computer interface based on mental tasks, J. Neural Eng., 8 (2011) 046014. [38] M.V.M. Yeo, X.P. Li, K.Q. Shen, E.P.V. Wilder-Smith, Can SVM be used for automatic EEG detection of drowsiness during car driving?, Safety Sci, 47 (2009) 115-124.

[39] S.L. Joutsiniemi, S. Kaski, T.A. Larsen, Self-organizing map in recognition of topographic patterns of EEG spectra, IEEE transactions on bio-medical engineering, 42 (1995) 1062-1068. 


\section{Figure legends:}

Fig. 1. The structure of the automatic detection system for distracted driving. Two models are proposed in this $\mathrm{BCl}$ system. The first model can remove the artifact signals and identify spatial brain sources from the raw EEG data. The data from the selected sources are the features for the second model. In the second model, the signature of concentrated or distracted driving is analyzed and detected.

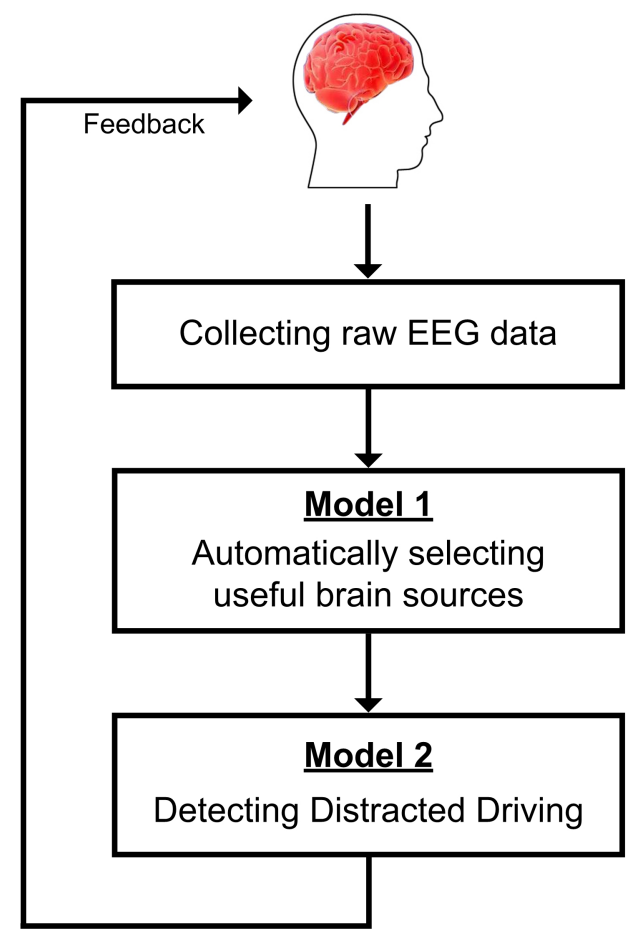

Fig. 2. Flowchart of the first model: the automatic selection of useful brain sources. Two layers are combined in this model. In layer 1, SVMRBF and 10 -fold cross-validation are applied to remove artifact component. The outputs of layer 1 are the useful components without artifacts. These selected components are then classified into distinct spatial brain regions according to the SOM in layer 2. 


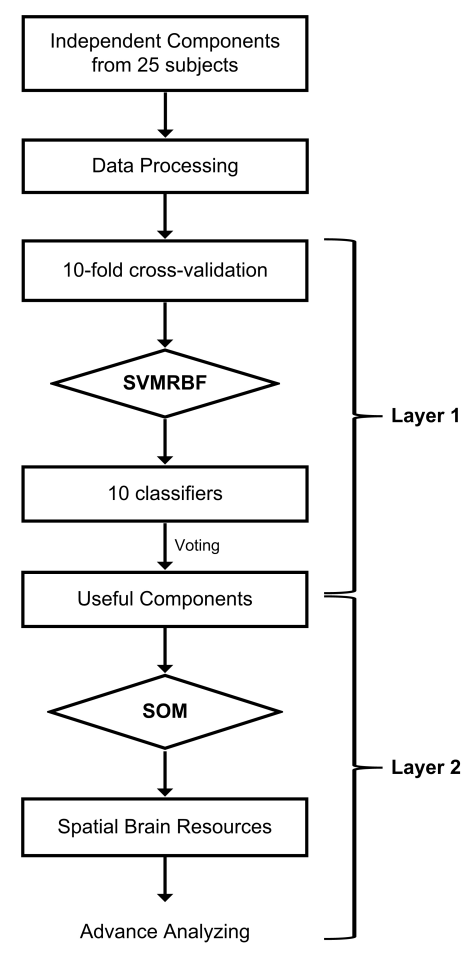

Fig. 3. Flowchart of the process used to detect distracted or concentrated driving. The EEG epochs of distracted driving are determined according to a majority vote. The leave-one-out method is also considered to verify the performance of the system.

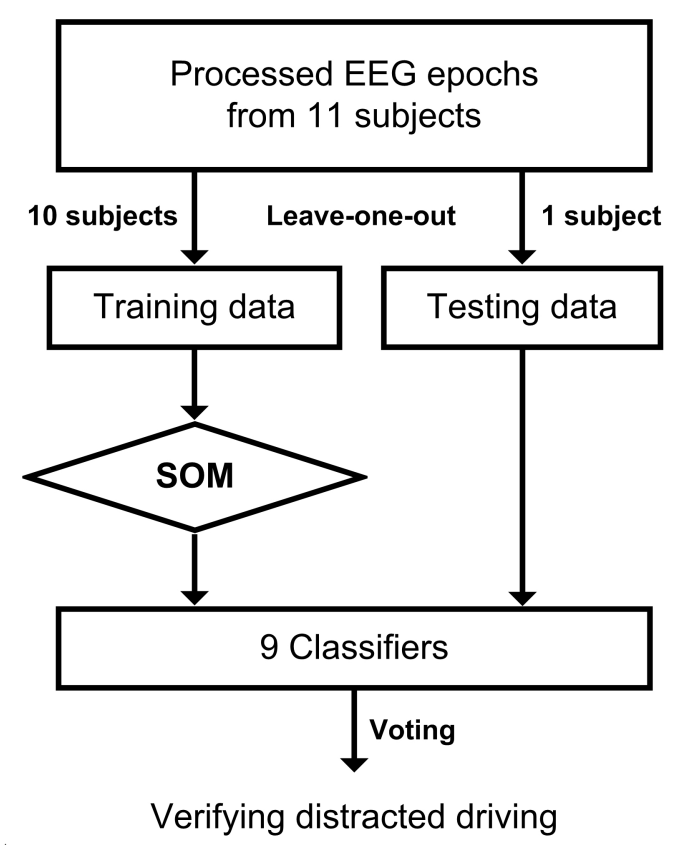

Fig. 4. All labeled components in one subject. (a) Each component is first classified as a useful component (U.C.) or artifact component (A.C.). (b) All useful components are then identified as frontal, central, parietal, occipital, left, 
or right motor components through distinct brain sources.

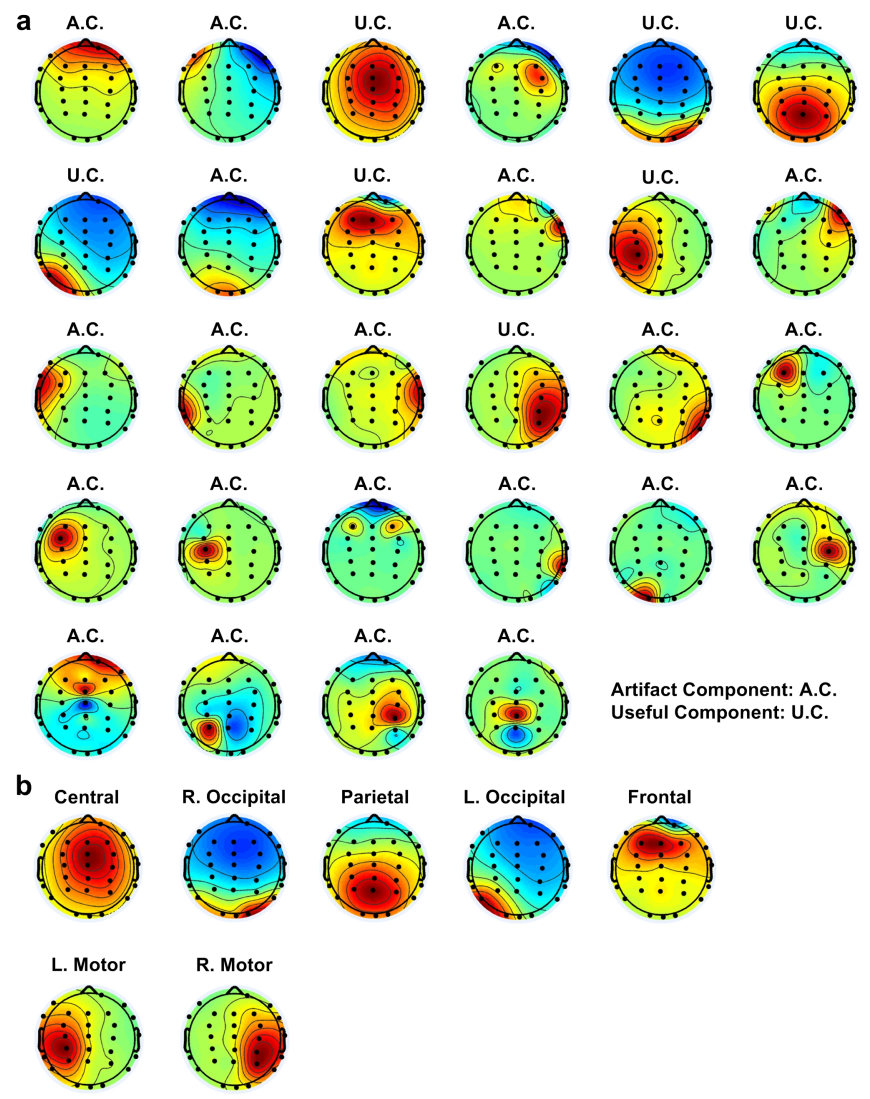

Fig. 5. The clustering results of distinct spatial components. The different brain sources are represented in various colors. Six brain sources are depicted in this map: the frontal, central, parietal, occipital, right motor, and left motor components.

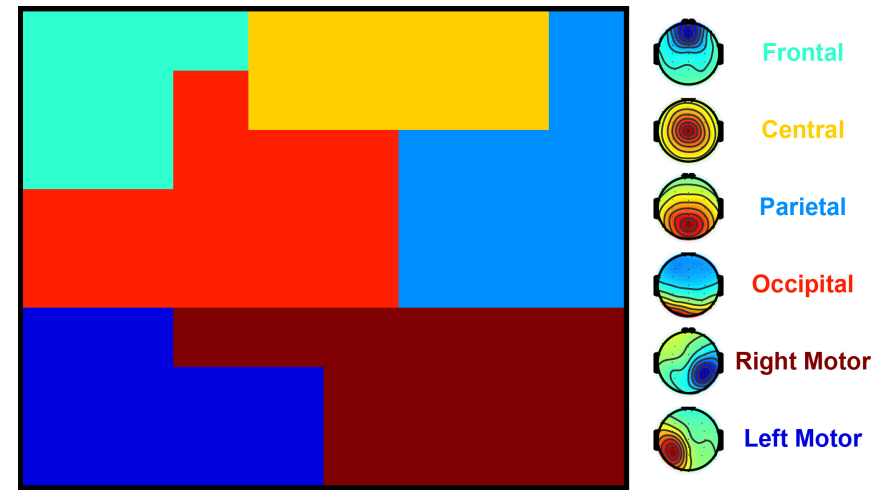

Fig. 6. The clustering results of the EEG epochs with five cases by selected brain sources. The EEG epochs of five cases are clustered, and the EEG data are extracted from five different brain sources: (a) frontal; (b) central; (c) parietal; (d) left motor; (e) right motor. 

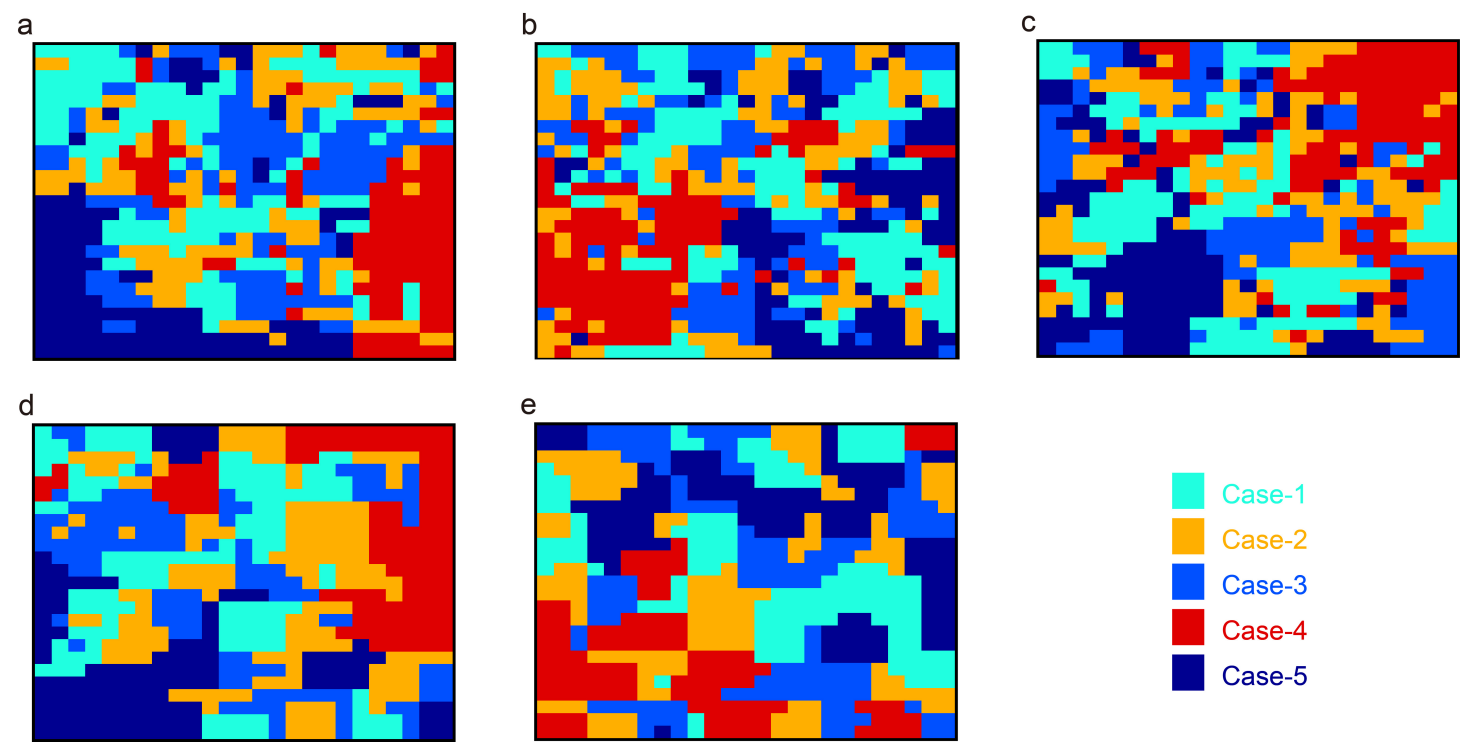

Fig. 7. Final results of clustering into four or five cases. The features of EEG epochs are composed of two brain sources: the frontal and left motor components. (a) Four conditions, excluding Case-2, are clustered. (b) The EEG epochs of all five cases are depicted.
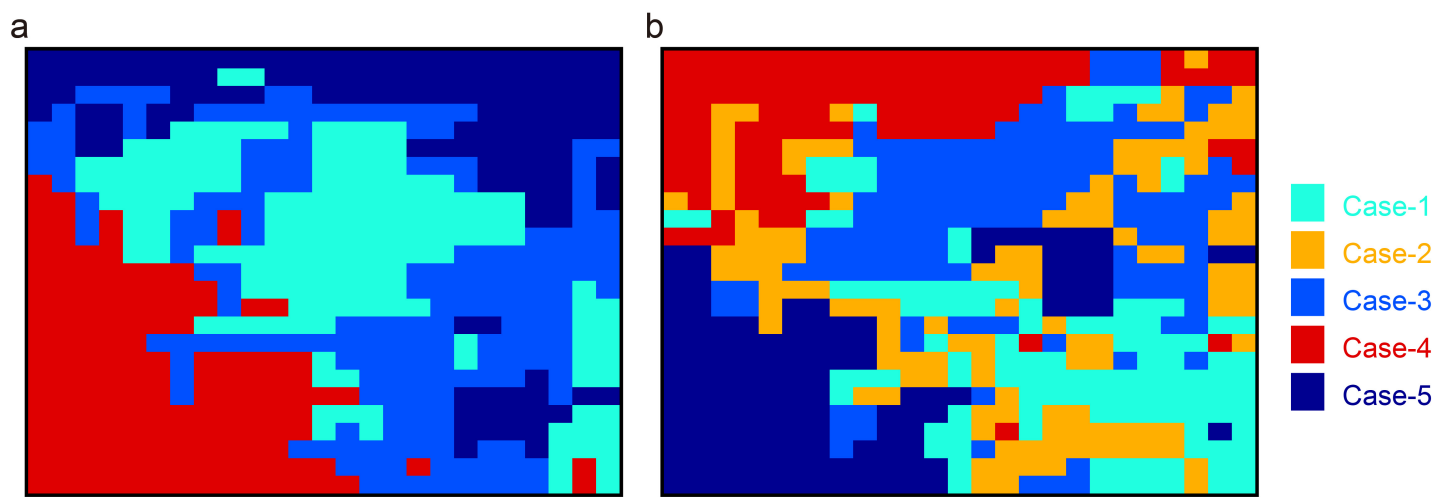

Table I: the number of each kind of independent components

\begin{tabular}{ccccccc}
\hline & Frontal & Central & Parietal & Occipital & L Motor & R Motor \\
\hline Numbers & 15 & 15 & 24 & 19 & 22 & 20 \\
\hline
\end{tabular}

Table 2 the accuracy and useful number in each subject

\begin{tabular}{ccccccccccccccc}
\hline & S1 & S2 & S3 & S4 & S5 & S6 & S7 & S8 & s9 & S10 & S11 & S12 & S13 & S14 \\
\hline Accuracy & 92.9 & 89.3 & 89.3 & 96.4 & 82.1 & 96.4 & 92.9 & 89.3 & 92.9 & 96.4 & 92.9 & 100 & 96.4 & 92.9 \\
Useful & 6 & 7 & 6 & 7 & 4 & 5 & 7 & 6 & 7 & 7 & 4 & 6 & 8 & 5 \\
\hline
\end{tabular}


Table 3 the recognized number of different brain sources

\begin{tabular}{ccccccc}
\hline & Frontal & Central & Parietal & Occipital & L. Motor & R. Motor \\
\hline Numbers & 14 & 10 & 9 & 9 & 11 & 10 \\
\hline
\end{tabular}

Table 4 the accuracy of each type of component

\begin{tabular}{lccccccc}
\hline & \multicolumn{3}{c}{ Dual Conditions } & & \multicolumn{2}{c}{ Single Conditions } \\
\cline { 2 - 4 } \cline { 6 - 7 } \cline { 6 - 7 } & Case-1(MID) & Case-2(D\&M) & Case-3(MID) & & Case-4(M) & Case-5(D) \\
\hline Frontal & $89.1 \pm 0.46$ & $89.3 \pm 4.1$ & $89.2 \pm 1.4$ & & $87.6 \pm 1.8$ & $95.7 \pm 2.1$ \\
Central & $76.8 \pm 3.9$ & $68.6 \pm 3.1$ & $68.9 \pm 1.0$ & & $75.1 \pm 2.5$ & $70.6 \pm 1.8$ \\
Parietal & $84.6 \pm 2.7$ & $73.8 \pm 1.8$ & $81.3 \pm 0.8$ & & $82.9 \pm 1.5$ & $76.9 \pm 2.4$ \\
L Motor & $96.2 \pm 0.9$ & $97.7 \pm 1.0$ & $97.3 \pm 0.9$ & & $96.7 \pm 1.6$ & $98.8 \pm 0.2$ \\
R Motor & $87.5 \pm 5.2$ & $83.5 \pm 9.8$ & $84.4 \pm 7.9$ & & $92.1 \pm 4.6$ & $92.4 \pm 3.9$ \\
\hline
\end{tabular}

Table 5: average accuracy of each map with combined features

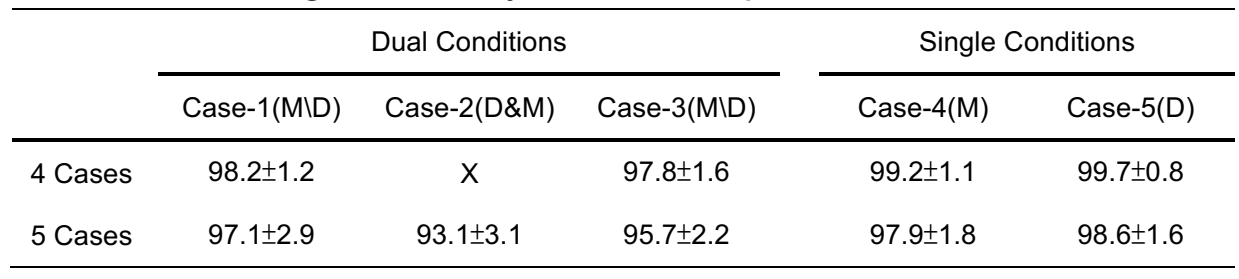

Table 6: average performance of recognizing EEG samples

\begin{tabular}{ccc} 
& Distracted Driving & Non-distracted Driving \\
\hline Validation & $84.1 \pm 1.8$ & $91.5 \pm 0.7$ \\
\hline
\end{tabular}

